\title{
Caracterización del estado ecológico de ríos mediterráneos ibéricos mediante el índice IBMWP (antes BMWP').
}

\author{
Javier Alba-Tercedor ${ }^{1}$, Pablo Jáimez-Cuéllar ${ }^{1}$, Maruxa Álvarez ${ }^{2}$, Juan Avilés ${ }^{3}$, Núria Bonada ${ }^{4}$, \\ Jesús Casas ${ }^{6}$, Andrés Mellado ${ }^{5}$, Manuel Ortega ${ }^{6}$, Isabel Pardo $^{2}$, Narcís Prat $^{4}$, Maria Rieradevall $^{4}$, \\ Santiago Robles ${ }^{3}$, Carmen Elisa Sáinz-Cantero ${ }^{1}$, Antonino Sánchez-Ortega ${ }^{1}, \mathrm{M}^{\mathrm{a}}$ Luisa Suárez $^{5}$, \\ Manuel Toro ${ }^{3}, \mathrm{M}^{\mathrm{a}}$ Rosario Vidal-Abarca ${ }^{3}$, Soledad Vivas ${ }^{6}$ y Carmen Zamora-Muñoz ${ }^{1}$
}

${ }^{1}$ Departamento de Biología Animal y Ecología. Universidad de Granada. Campus Universitario de Fuentenueva. 18071 Granada.

2 Área de Ecología. Universidad de Vigo. Campus Lagoas-Marcosende. 36200 Vigo.

${ }^{3}$ CEDEX. División de Ecología de los Sistemas Acuáticos Continentales. Paseo Bajo Virgen del Puerto, 3. 28005 Madrid.

${ }^{4}$ Departament d'Ecologia. Universitat de Barcelona. Diagonal, 645. 08028 Barcelona.

${ }^{5}$ Departamento de Ecología e Hidrología. Universidad de Murcia. Campus de Espinardo. 30100 Murcia.

${ }^{6}$ Departamento de Biología Vegetal y Ecología. Universidad de Almería. Cañada de San Urbano, s/n. 04120

Almería.

\section{RESUMEN}

En base a los datos obtenidos a lo largo de la primera fase del proyecto GUADALMED (1999-2001), se estudia la calidad biológica de las aguas de 65 ríos de 12 cuencas mediterráneas ibéricas y el comportamiento del índice IBMWP ("Iberian Biomonitoring Working Party"; antes BMWP'). Se proponen unas bases para el establecimiento de las clases de calidad para la evaluación de estado ecológico de los ríos, siguiendo las directrices de la Directiva Marco del Agua, basadas en los valores del índice obtenidos en las estaciones de referencia de cada una de las tipologías de ríos obtenidas en el estudio. Tras la comparación entre las cinco clases originales del índice y las posibles nuevas acotaciones obtenidas se observó una total coincidencia para todos los casos en que se contaba con un número de estaciones de referencia elevado (>50), observándose discrepancias respecto a aquellas tipologías de ríos en que el número de estaciones era escaso $(<50)$. Por lo se propone seguir utilizando las mismas acotaciones del IBMWP hasta no contar con un mayor número de estaciones de referencia que permita evaluar si efectivamente las discrepancias observadas se deben a características diferenciales de las diferentes tipologías de ríos, o por el contrario son el resultado de no disponer de una representación suficiente de estaciones de referencia en algunas de las tipologías.

Palabras clave: clasificación, estado ecológico, ríos mediterráneos, España, Portugal, IBMWP.

\begin{abstract}
Using baseline data obtained during the first phase (1999-2001) of the project GUADALMED, we examined the applicability of the IBMWP (Iberian Bio-monitoring Working Party; former BMWP') to assess the quality of 65 rivers and streams within 12 Iberian Mediterranean basins. Moreover, criteria for the establishment of classes to assess the ecological status of the Iberian Mediterranean rivers are proposed. Criteria followed the European Water Framework Directive, and used the index values found in the reference stations of each river type in the study. By comparing the five original classes of the IBMWP index and the candidate new classes, similar values were obtained in all cases when the number of reference stations was high ( $>50)$, while discrepancies were observed within river types when the number of stations was low ( $<50)$. Therefore, we propose that the same IBMWP classes continue to be used, until a greater number of reference stations are available, which would be necessary to evaluate whether discrepancies observed are due to specific differences of river types, or are due to an insufficient representation of reference stations of some river types.
\end{abstract}

Key words: classification, ecological status, mediterranean rivers, Spain, Portugal, IBMWP. 


\section{INTRODUCCIÓN}

La experiencia y uso de métodos biológicos para evaluar la calidad de las aguas de los ríos es amplia y de gran tradición en la Península Ibérica (AlbaTercedor \& Prat, 1992). Tanto en España, como en Portugal, en la última década se ha extendido de forma espectacular el uso de una adaptación del sistema británico de puntuación BMWP (Armitage et al., 1983), conocida como índice BMWP' (AlbaTercedor \& Sánchez-Ortega, 1988).

El proyecto coordinado GUADALMED ha supuesto una oportunidad única para evaluar la situación de diferentes tipos de ríos situados a lo largo del arco mediterráneo ibérico (incluidos los cursos de agua de las Islas Baleares), así como banco de trabajo para testar métodos de evaluación como paso previo al establecimiento del estado ecológico de los ríos que demanda la aprobación de la Directiva Marco del Agua (D.O.C.E., 2000). Asimismo, dado el amplio uso del índice BMWP' y que han transcurrido muchos años desde su publicación, se hace necesaria una revisión del mismo y de las adiciones posteriores (AlbaTercedor, 1996; Alba-Tercedor \& Pujante, 2000). Además se hace imprescindible una evaluación de su acotación en clases de calidad de acuerdo con los criterios que fija la nueva directiva marco sobre el agua (op. cit.).

En el presente trabajo analizamos la situación de la calidad de las aguas de 12 cuencas mediterráneas ibéricas y el comportamiento del índice BMWP', proponiendo unas bases para el establecimiento de las clases de calidad. Todo ello en base a los datos obtenidos a lo largo de la primera fase del proyecto GUADALMED (1999-2001). Asimismo, y según el acuerdo obtenido durante la presentación de estos datos en el III Congreso ibérico de Limnología (Alba-Tercedor et al., 2002), proponemos que a partir de ahora el BMWP' pase a llamarse IBMWP ("Iberian Biomonitoring Working Party").

\section{MATERIAL Y MÉTODOS}

Como paso previo, al inicio del estudio se llevó a cabo un ejercicio de intercalibración metodo- lógica en que se testaron dos protocolos de muestreo correspondientes a los índices FBILL (Prat et al., 2000) e IBMWP (Alba-Tercedor \& Sánchez-Ortega, 1988; Alba-Tercedor, 1996; Alba-Tercedor \& Pujante, 2000). En base a los resultados se seleccionó el método IBMWP (ver Bonada et al. (a), en este volumen). Se estudiaron 65 cursos de agua pertenecientes a 12 cuencas hidrográficas mediterráneas. En ellas se muestrearon estacionalmente, a lo largo de dos ciclos anuales (de Abril 1999 a Marzo de 2001) un total de 157 estaciones de muestreo (ver Robles et al., en este volumen).

En una primera fase se seleccionaron estaciones de referencia en los diferentes tramos de las cuencas siguiendo el criterio de que fuesen las que mejores condiciones tuviesen (Reynoldson $\&$ Wright, 2000). Pero la dificultad o en muchos casos imposibilidad material de encontrar en buenas condiciones puntos en los tramos medios y bajos, hizo que en una segunda fase se tomaran criterios mas restrictivos que permitieran asegurar un buen estado ecológico de las mismas. De tal forma que como condiciones de referencia se utilizaron tan solo los datos provenientes de esta segunda fase (ver Bonada et al. (c), en este volumen).

Además del muestreo de los macroinvertebrados, se analizaron diferentes parámetros físicoquímicos (ver Toro et al., en este volumen), y se evaluó tanto la calidad del bosque de ribera mediante el índice QBR (Munné et al., 1988; ver Suárez et al., en este volumen), como del hábitat fluvial (ver Pardo et al., en este volumen). Con los resultados se generó una base de datos global que es la que se ha utilizado como base para este trabajo.

Para determinar que parámetros físico-químicos explicaban mejor las variaciones del IBMWP se llevó a cabo un análisis de correlación simple ( $\mathrm{R}$ de Pearson), así como un análisis de regresión múltiple por pasos (Dixon \& Jennrich, 1983; Edwards, 1985; Sokal \& Rohlf, 1979), método que ha sido utilizado para los mismos propósitos en otros trabajos similares (Armitage et al., 1983; Moss et al., 1987; Rodríguez \& Wright, 1988; 1989; Zamora-Muñoz et al., 1995; entre otros). 

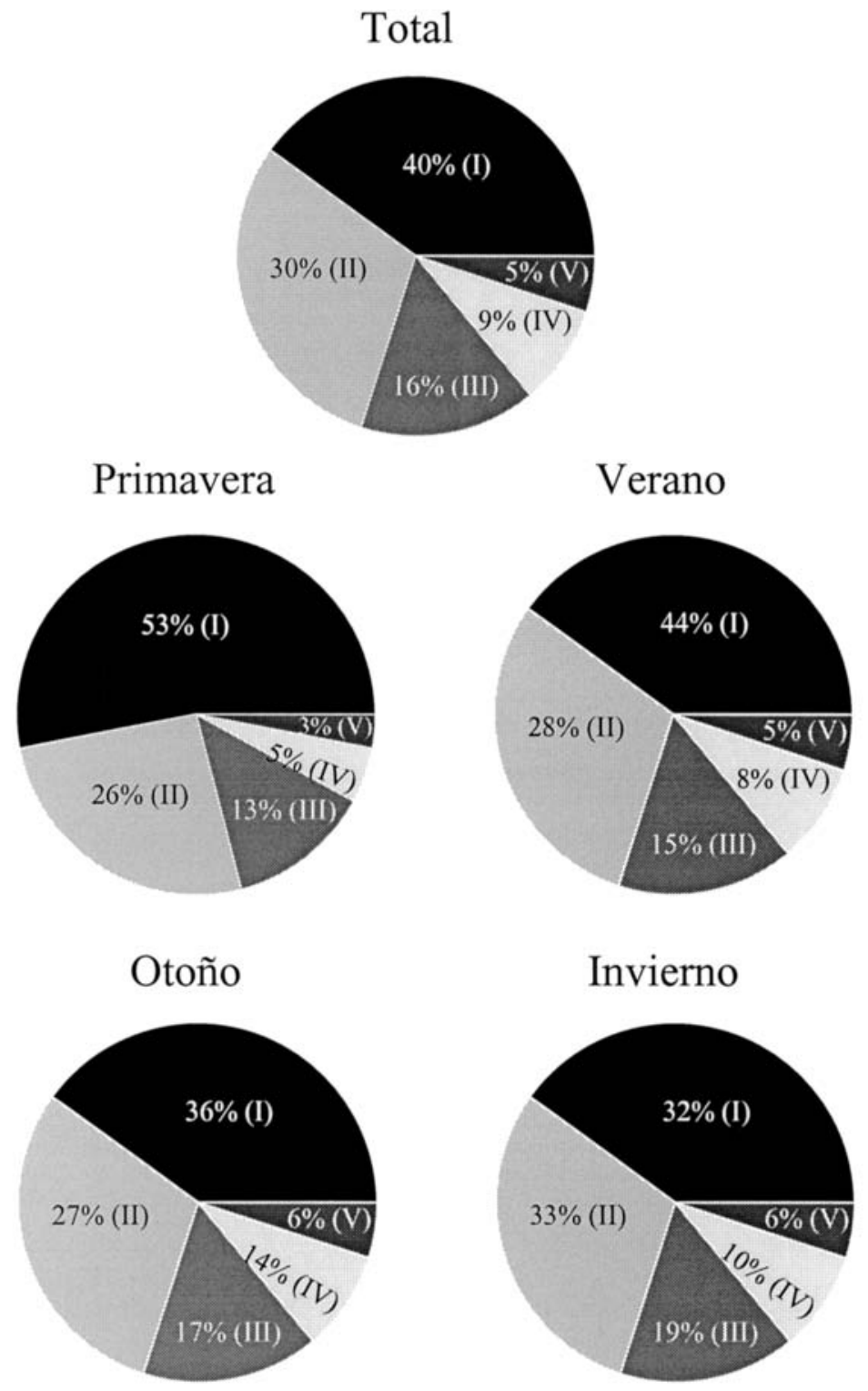

Figura 1. Situación global de la calidad biológica de las aguas y variaciones estacionales del conjunto de puntos de muestreo estudiados. Entre paréntesis se presenta la clase de calidad que corresponde a cada sector (I = Buena; II = Aceptable; III = Dudosa; IV = Crítica; V = Muy crítica — ver Jáimez-Cuéllar, este volumen). Overall situation of the water biological quality and seasonal variations of this at the sampling sites studied. Quality classes are presented in brackets $(I=$ Good; $I I=P a s s a b l e ; I I I=D u b i o u s$; $I V=$ Critical; $V=$ Very critical —see Jáimez-Cuéllar, this issue)

Como variable dependiente se utilizó el IBMWP y como variables independientes los parámetros físicos (altitud, pendiente, caudal, temperatura, distancia al origen, índice de hábitat y QBR) y químicos (sólidos en suspensión, amonio, nitra- tos, nitritos, fosfatos, sulfatos, cloruros, oxígeno, $\mathrm{pH}$ y conductividad). Antes de realizar los análisis, siguiendo a Digby \& Kempton (1987), las variables físico-químicas se transformaron para normalizarlas. 


\section{RESULTADOS}

Analizando los datos en su conjunto y siguiendo las clases de calidad originales, establecidas para los valores del índice IBMWP por AlbaTercedor \& Sánchez-Ortega (1988), el 70\% de las estaciones de muestreo presentaron aguas en situación entre "buena" y "aceptable" (clases I y
II), existiendo ligeras variaciones estacionales (Fig. 1). Una representación global de la situación general, expresada como valores medios del índice IBMWP, de las diferentes cuencas se puede observar en la figura 2 .

Al estudiar la distribución estacional de las clases de calidad, comparando las estaciones de referencia con las de no referencia (Fig. 3), se

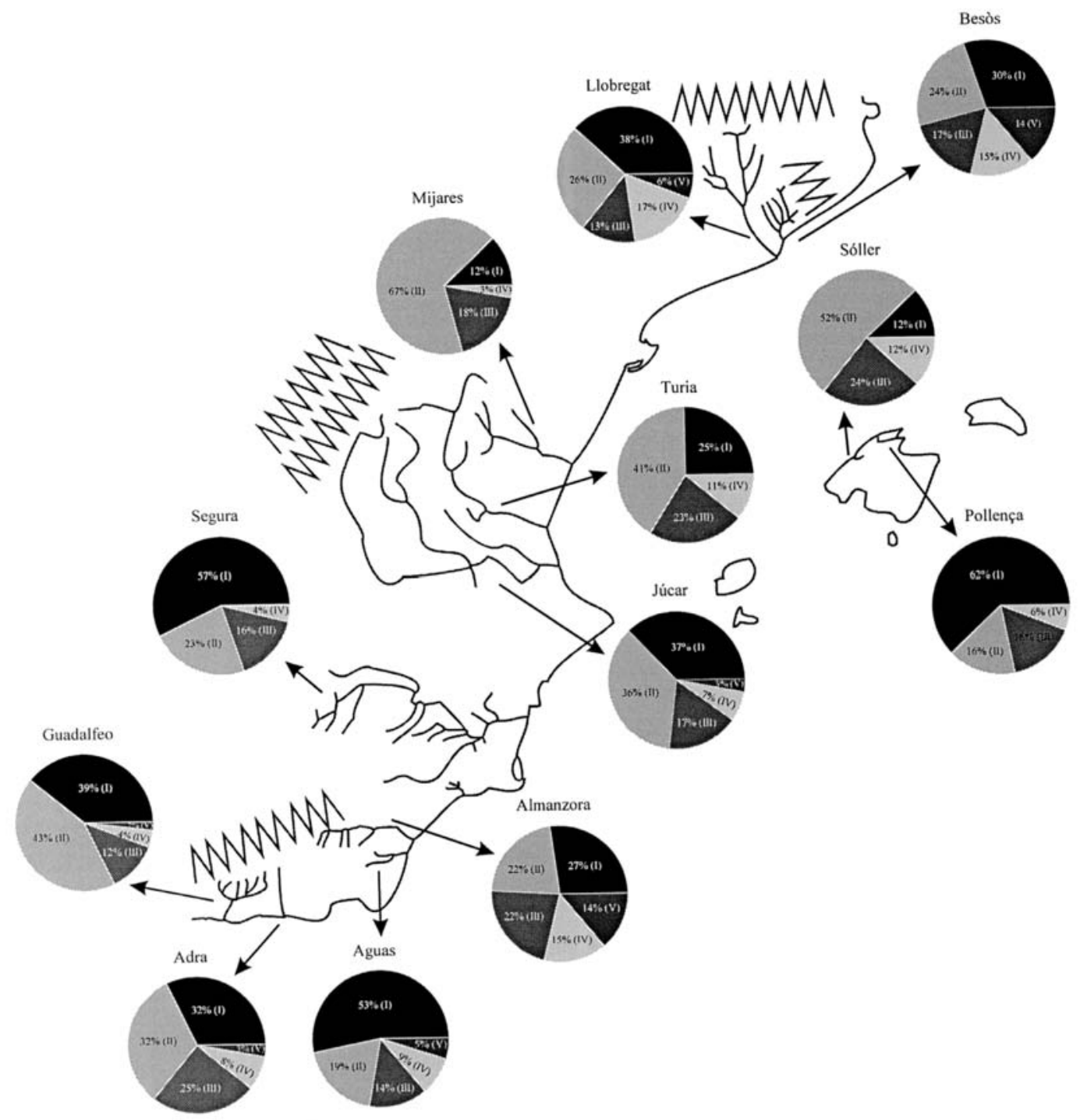

Figure 2. Calidad biológica media de las diferentes cuencas estudiadas a lo largo del arco mediterráneo ibérico. Entre paréntesis se presenta la clase de calidad que corresponde a cada sector ( $\mathrm{I}=$ Buena; II = Aceptable; III = Dudosa; IV = Crítica; V = Muy crítica — ver Jáimez-Cuéllar, este volumen). Average situation of the water biological quality of the mediterranean different river basins studied along the Iberian Mediterranean coast. Quality classes presented in brackets $(I=$ Good; $I I=P a s s a b l e ; I I I=D u b i o u s$; IV = Critical; $V=$ Very critical —see Jáimez-Cuéllar, this issue) 
Tabla 1. Correlaciones entre los diferentes parámetros físicoquímicos y el índice IBMWP. Nota: La letra " $T$ " antes del nombre de la variable significa que ésta ha sido transformada para asemejarla a una distribución Normal: T-IBMWP $(\mathrm{X}=$ $\left.\log _{10}(\mathrm{X}+3)\right)$; T-QBR $\left(\mathrm{X}=\mathrm{X}^{1 / 2}\right)$; T-Nitratos $\left(\mathrm{X}=\log _{10}(\mathrm{X}+\right.$ $0.3)$ ); T-Sulfatos $\left(\mathrm{X}=\log _{10}(\mathrm{X}+0.8)\right)$; T-Cloruros $\left(\mathrm{X}=\log _{10}\right.$ $(\mathrm{X}+0.6))$; T-Caudal $\left(\mathrm{X}=\log _{10}(\mathrm{X})\right)$; T-Oxígeno $\left(\mathrm{X}=\mathrm{X}^{1 / 2}\right)$; T$\mathrm{T}^{\mathrm{a}}$ del agua $\left(\mathrm{X}=\log _{10}(\mathrm{X}+5)\right)$; T-Conductividad $\left(\mathrm{X}=\log _{10}\right.$ $(\mathrm{X}+5))$; T-Altitud $\left(\mathrm{X}=\log _{10}(\mathrm{X}+5)\right)(* \mathrm{p}<0.05 ; * * \mathrm{p}<0.025$; $\mathrm{p}<0.001)$. Correlation coefficients between the physico-chemical variables and the IBMWP index. Note: The letter " $T$ " before the name of the variable means it has been transformed to fulfil the normality requirement: $T-I B M W P\left(X=\log _{10}\right.$ $(X+3)) ; T-Q B R\left(X=X^{1 / 2}\right) ;$ T-Nitrates $\left(X=\log _{10}(X+0.3)\right) ; T$ Sulphates $\left(X=\log _{10}(X+0.8)\right) ;$ T-Chloride $\left(X=\log _{10}\right.$ $(X+0.6)) ;$ T-Discharge $\left(X=\log _{10}(X)\right)$; T-Oxygen $\left(X=X^{1 / 2}\right) ; T-$ $T^{a}$ of water $\left(X=\log _{10}(X+5)\right) ;$ T-Conductivity $\left(X=\log _{10}\right.$ $(X+5))$; T-Altitude $\left(X=\log _{10}(X+5)\right)(* p<0.05$; ** $p<0.025$; $p<0.001)$

\begin{tabular}{ll}
\hline & T-IBMWP \\
\hline IHF & $0.4620 * * *$ \\
T-QBR & $0.7048 * * *$ \\
SS & -0.0713 \\
Amonio & $-0.5020^{* * *}$ \\
Nitritos & $-0.1584 *$ \\
T-Nitratos & -0.0815 \\
Fosfatos & $-0.3225 * * *$ \\
T-Sulfatos & $-0.4214 * * *$ \\
T-Cloruros & $-0.6301 * * *$ \\
T-Caudal & -0.1161 \\
T-Oxígeno & 0.0944 \\
pH & $-0.1673 *$ \\
T-T del agua & $-0.2425 * *$ \\
T-Conductividad & $-0.5310^{* * *}$ \\
T-Altitud & $0.6540^{* * *}$ \\
\hline
\end{tabular}

observa que tal y como cabía esperar, la mayoría de las estaciones de referencia presentan una buena calidad de sus aguas. Sin embargo, en la mayoría de las cuencas existen estaciones de muestreo que fueron consideradas como de referencia en la primera fase, y que claramente sufren algún tipo de alteración importante (clases de calidad III).

La degradación de las riberas que sufren los ríos mediterráneos desde su cabecera, y el progresivo incremento de vertidos a sus aguas, se refleja claramente en la alta correlación positiva que muestra el IBMWP tanto con la altitud como con la calidad del hábitat fluvial (Tabla 1, y Pardo et al., en este volumen), conservación del bosque de ribera (Tabla 1 y Suárez et al., en este volumen) y negativa con parámetros químicos que denotan contaminaciones, tales como el amonio, nitritos y fosfatos (Tabla 1). De hecho, para determinar cuales de esos parámetros explican mejor la variación del índice biológico, se realizó un análisis de regresión múltiple por pasos. Los resultados mostraron que un $63 \%$ de la varianza del IBMWP fue explicado, de forma altamente significativa, por tres parámetros (Tabla 2): QBR y altitud, de forma positiva, y amonio negativamente. En un trabajo anterior Zamora-Muñoz et al. (1995) encontraron resultados similares en cuanto a la variación del índice. El resto de la varianza es explicado por los macroinvertebrados (Vivas et al., en este volumen).

Tabla 2. Resultados de la regresión múltiple por pasos entre el índice IBMWP y los parámetros físico-químicos medidos. Nota: Unidades: QBR: Adimensional, Amonio (mg/l), Altitud (m.s.n.m.). El análisis se ha realizado con una tolerancia $=0.4$. Results of the multiple stepwise regression between the index IBMWP and physico-chemical variables measured. Nota: Units: QBR: unitless, Ammonium (mg/l), Altitude (m.a.s.l.). Tolerance $=0.4$.

Variable Dependiente: T-IBMWP

Múltiple R: 0.7932

Múltiple $\mathrm{R}^{2}: 0.6292$

$\mathrm{R}^{2}$ Ajustada: 0.6224

$\mathrm{F}(3,165)=93.33 ; \mathrm{p}<0.00001$

Intercepción: 0.0772

\begin{tabular}{lrrrcrl}
\hline & Variable & Beta & S.E. & B S.E. de B & t (165) & p $=$ \\
\hline T-QBR & 0.3424 & 0.0730 & 0.3025 & 0.0645 & 4.6921 & 0.000006 \\
Amonio & -0.3223 & 0.0507 & -0.2967 & 0.0467 & -6.3534 & 0.000000 \\
T-Altitud & 0.3457 & 0.0697 & 0.3511 & 0.0708 & 4.9574 & 0.000002 \\
\hline
\end{tabular}


Acotación de los valores del IBMWP como expresión del estado ecológico de los ríos mediterráneos

Según la DMA, independientemente del método utilizado para la evaluación del estado ecológico, éste ha de medirse como desviación del muy buen estado ecológico, el cual corresponde a las condiciones de referencia aplicables a cada masa de agua. Han de considerarse cinco clases del estado ecológico: "muy bueno", "bueno”, “aceptable", "deficiente" y "malo". A cada una de las cuales se le asignará respectivamente uno de los siguientes colores: azul, verde, amarillo, naranja y rojo. Si bien, como señala Ortiz (en este volumen) ha sido un error la permanencia del término aceptable, manteniendo la traducción de "fair" de una primera versión del texto en inglés, sin
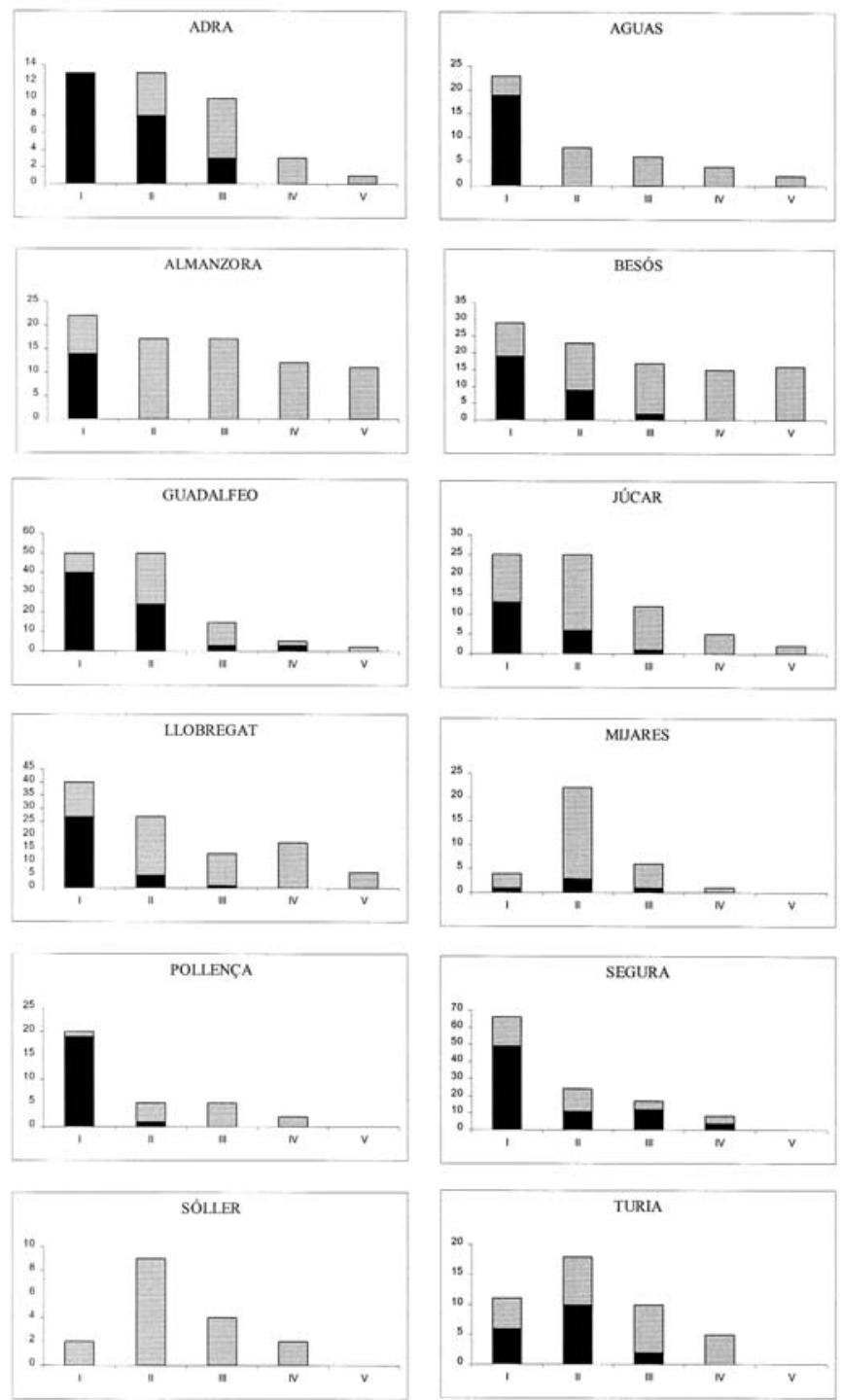

Referencia

No-Referencia

Figura 3. Distribución estacional de las clases de calidad biológica del agua, comparando las estaciones de referencia con las de no referencia. Seasonal distribution of the biological water quality classes, comparing reference and non-reference stations. 
que en la versión castellana se incorporara el término "moderado" ("moderate") que fue el que definitivamente quedó en la versión inglesa.

Comparando los requisitos de división del estado ecológico en cinco clases de calidad, así como el código de colores que a cada uno de ellos hace corresponder la DMA, es fácil caer en la tentación de hacer una transposición inmediata de las clases de calidad establecidas originariamente para el IBMWP (Alba-Tercedor \& Sánchez-Ortega, 1988), ya que este índice divide la calidad de las aguas en cinco clases a los que le asigna los mismos códigos de color que contempla la DMA, y en principio bastaría con cambiar la terminología de las clases de calidad de este índice ("buena", "aceptable", "dudosa", "crítica" y "muy crítica") por la de la DMA. Pero con ello no cumpliríamos las exigencias de la nueva legislación que exige el establecimiento de las clases de calidad en función del grado de desviación de las condiciones de referencia. Además, surge la duda sobre la validez de aplicación en todas las tipolo- gías de ríos ibéricos de las clases de calidad establecidas para los valores del IBMWP. De hecho, ha habido autores que han propuesto unos ligeros ajustes para determinados ríos (Rico et al., 1992).

Existen diferentes formas de establecer intervalos de clases para los valores de los índices biológicos que reflejen el grado de separación respecto de las condiciones de referencia, y que por tanto permitan definir en base a ellos clases del estado ecológico. Siguiendo una metodología similar a la de Barbour et al. (1996; 1999) nosotros seguimos el siguiente proceso: tras hacer agrupaciones de los ríos en tipologías (ecotipos) (ver Bonada et al., (b) en este volumen), se establecen estaciones de referencia en cada uno de los mismos (ver Bonada et al., (c) en este volumen) y se estudia el universo de valores del índice biológico en cada tipología de curso de agua, estableciendo las clases de calidad en función del grado de desviación respecto a los datos obtenidos en las estaciones de referencia. Así para cada tipo se establecen las

Tabla 3. Valores del IBMWP en las diferentes tipologías de ríos mediterráneos estudiados. En la parte superior (a) se incluyen los valores obtenidos en todas las estaciones de muestreo. En la parte inferior (b) se han considerado tan solo aquellas estaciones de muestreo que cumplen los criterios de referencia. Values of the IBMWP index in the different typologies of Mediterranean rivers studied. Top, (a) values of IBMWP obtained in all sampling stations. Bottom, (b) only sampling stations complying with the reference criteria are included.

\begin{tabular}{|c|c|c|c|c|c|c|c|c|}
\hline $\mathrm{a}$ & $\mathbf{n}$ & Media & Mediana & Mínimo & Máximo & P25 & P75 & Dev. St. \\
\hline CAL/CAB & 91 & 130.8 & 126 & 2 & 272 & 84 & 175 & 64.88 \\
\hline CAL/GB & 32 & 78.7 & 71.5 & 43 & 135 & 62 & 88.5 & 25.58 \\
\hline CAL/MB & 233 & 82.5 & 75 & 2 & 248 & 49 & 111 & 45.01 \\
\hline KARST & 60 & 151 & 141 & 59 & 251 & 122 & 170.5 & 46.36 \\
\hline LLANURA & 4 & 15.8 & 14 & 8 & 27 & 9.5 & 22 & 8.38 \\
\hline RAMBLAS & 81 & 73.5 & 78 & 5 & 151 & 50 & 100 & 31.99 \\
\hline SIL/CAB & 103 & 108.8 & 110 & 7 & 265 & 81 & 135 & 42.63 \\
\hline SIL/MB & 140 & 53 & 48 & 0 & 164 & 25.5 & 77.5 & 34.96 \\
\hline TEMPORAL & 48 & 90.3 & 89 & 33 & 178 & 61.5 & 110 & 34.1 \\
\hline $\mathrm{b}$ & $\mathbf{n}$ & Media & Mediana & Mínimo & Máximo & P25 & P75 & Dev. St. \\
\hline $\mathrm{CAL} / \mathrm{CAB}$ & 63 & 155.9 & 151 & 39 & 272 & 116 & 200 & 56.05 \\
\hline $\mathrm{CAL} / \mathrm{GB}$ & 8 & 72 & 73 & 47 & 90 & 67 & 79.5 & 12.94 \\
\hline $\mathrm{CAL} / \mathrm{MB}$ & 57 & 131.2 & 127 & 59 & 248 & 104 & 148 & 40.09 \\
\hline KARST & 59 & 152.3 & 141 & 59 & 251 & 123 & 172 & 45.61 \\
\hline LLANURA & 0 & & & & & & & \\
\hline RAMBLAS & 31 & 65.7 & 60 & 27 & 138 & 39 & 84 & 29.09 \\
\hline SIL/CAB & 60 & 129.4 & 130 & 65 & 265 & 109 & 146 & 35.19 \\
\hline SIL/MB & 32 & 78.5 & 76.5 & 19 & 148 & 62 & 91.5 & 29.40 \\
\hline TEMPORAL & 19 & 98 & 95 & 47 & 178 & 81 & 112 & 35.11 \\
\hline
\end{tabular}




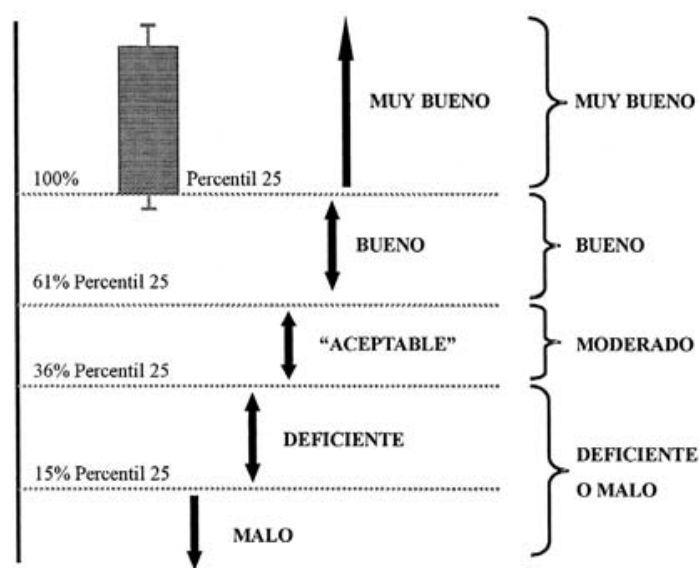

Figura 4. Propuesta de establecimiento de clases de estado ecológico, en función de los valores obtenidos del IBMWP (=BMWP') en las estaciones de referencia de cada tipo de río, siguiendo los criterios de la Directiva Marco del Agua. Proposal for the establishment of ecological status classes, on the basis of the values of IBMWP (=BMWP') obtained in the reference stations of each river type, in accordance with the criteria set out in the Water Framework Directive.

clases de calidad siguiendo los intervalos que cumplen las condiciones esquematizadas en la figura 4, considerando los porcentajes del per- centil 25 correspondientes al: $100 \%, 61 \%, 36 \%$ y $15 \%$, respectivamente, como los límites entre las cinco clases del estado ecológico. Así, en una primera aproximación, se calcularon los estadísticos mencionados en el conjunto de estaciones muestreadas (Tabla 3a). Posteriormente se calcularon los mismos teniendo en cuenta tan solo aquellas estaciones de muestreo que pasaron el filtro de las restricciones establecidas para considerarlas de referencia (Tabla 3b), y en base a los resultados obtenidos se hizo una aproximación tentativa al establecimiento de nuevas clases de calidad (Tabla 4).

\section{DISCUSIÓN}

\section{Problemas encontrados y necesidades de futuros estudios}

El mayor problema encontrado lo constituye la dificultad de encontrar "auténticas" estaciones de referencia, indispensables para poder discri-

Tabla 4. Tentativa de establecer acotaciones de los valores del índice IBMWP, para definir el estado ecológico de diferentes tipologías de cursos de agua mediterráneos de la Península Ibérica. Se indican las acotaciones de los valores originales establecidas por AlbaTercedor \& Sánchez-Ortega (1988) (Ver explicaciones adicionales en el texto). Attempt at establishing classes of values of the index IBMWP to define the ecological status of different types of Mediterranean rivers and streams of the Iberian Peninsula. The original classes established by Alba-Tercedor \& Sánchez-Ortega (1988) are indicated (for further details, see text).

\begin{tabular}{|c|c|c|c|c|c|}
\hline Rango & Clase & Originales & $\mathrm{Cal} / \mathrm{Cab}$ & $\mathbf{C a l} / \mathbf{G b}$ & $\mathrm{Cal} / \mathrm{Mb}$ \\
\hline $\begin{array}{l}\text { Muy bueno } \\
\text { Bueno } \\
\text { Moderado } \\
\text { Deficiente } \\
\text { Malo }\end{array}$ & $\begin{array}{l}\text { Clase I } \\
\text { Clase II } \\
\text { Clase III } \\
\text { Clase IV } \\
\text { Clase V }\end{array}$ & $\begin{array}{l}>100 \\
61-100 \\
36-60 \\
15-35 \\
<15\end{array}$ & $\begin{array}{l}>116 \\
71-115 \\
42-70 \\
17-69 \\
<17\end{array}$ & $\begin{array}{l}>67 \\
41-66 \\
24-40 \\
10-23 \\
<10\end{array}$ & $\begin{array}{l}>104 \\
63-103 \\
37-62 \\
16-36 \\
<16\end{array}$ \\
\hline Rango & Clase & Originales & Karst & Llanura & Ramblas \\
\hline $\begin{array}{l}\text { Muy bueno } \\
\text { Bueno } \\
\text { Moderado } \\
\text { Deficiente } \\
\text { Malo }\end{array}$ & $\begin{array}{l}\text { Clase I } \\
\text { Clase II } \\
\text { Clase III } \\
\text { Clase IV } \\
\text { Clase V }\end{array}$ & $\begin{array}{l}>100 \\
61-100 \\
36-60 \\
15-35 \\
<15\end{array}$ & $\begin{array}{l}>123 \\
75-122 \\
44-74 \\
18-43 \\
<18\end{array}$ & $\begin{array}{l}- \\
- \\
- \\
- \\
-\end{array}$ & $\begin{array}{l}>39 \\
24-38 \\
14-23 \\
6-13 \\
<6\end{array}$ \\
\hline Rango & Clase & Originales & Sil/Cab & Sil/Mb & Temporales \\
\hline $\begin{array}{l}\text { Muy bueno } \\
\text { Bueno } \\
\text { Moderado } \\
\text { Deficiente } \\
\text { Malo }\end{array}$ & $\begin{array}{l}\text { Clase I } \\
\text { Clase II } \\
\text { Clase III } \\
\text { Clase IV } \\
\text { Clase V }\end{array}$ & $\begin{array}{l}>100 \\
61-100 \\
36-60 \\
15-35 \\
<15\end{array}$ & $\begin{array}{l}>109 \\
66-108 \\
39-65 \\
16-38 \\
<16\end{array}$ & $\begin{array}{l}>62 \\
38-61 \\
22-37 \\
9-21 \\
<9\end{array}$ & $\begin{array}{l}>81 \\
50-80 \\
29-49 \\
12-28 \\
<12\end{array}$ \\
\hline
\end{tabular}


minar las clases del estado ecológico. De hecho, si analizamos atentamente las nuevas acotaciones obtenidas (Tabla 4) en esta primera fase, se observa que ninguna de las estaciones de muestreo situadas en la tipología de "llanura" cumplieron las condiciones fijadas como de referencia y de otras, como las situadas en cursos "temporales" y en los "calcáreos de cabecera" ("Cal/Cab"), fue muy bajo el número de estaciones de muestreo que cumplieran las condiciones de referencia, en base a las cuales se han hecho este primer intento de establecimiento de nuevas acotaciones del índice. A pesar de ello resulta muy interesante el comprobar la casi total coincidencia existente entre las acotaciones originales de calidad del IBMWP (=BMWP') (AlbaTercedor \& Sánchez-Ortega, 1988) y las nuevas acotaciones obtenidas para las tipologías de ríos en donde se contaba con un número de estaciones de referencia mayor de 50 (cabeceras de ríos calcáreos, cursos medios y bajos de ríos calcáreos, Cal/MB, Karst, y los ríos silíceos de cabecera). Sin embargo existen unos fuertes cambios respecto a los intervalos de clases originales en el resto de casos en donde el número de estaciones de referencia disponibles para hacer las nuevas acotaciones fue pequeño $(<50)$. Por ello consideramos que para el establecimiento $\mathrm{y} / \mathrm{o}$ validación de posibles nuevos intervalos que definan las clases del estado ecológico de los diferentes tipos de ríos mediterráneos es necesaria una segunda etapa, en la que se disponga de un mayor número de estaciones de referencia. Lo cual esperamos poder solucionar en la segunda fase del proyecto, y con el desarrollo de un método predictivo (MEDPACS). Por ello, hasta no poder contar con un mayor número de estaciones de referencia que permita evaluar si efectivamente las discrepancias observadas se deben a características diferenciales de las diferentes tipologías de ríos, o por el contrario son el resultado de no disponer de una representación suficiente de estaciones de referencia en algunas de las tipologías, consideramos que por el momento deben seguir utilizándose las acotaciones originales del índice IBMWP para establecer las cinco clases del estado ecológico de los ríos.

\section{AGRADECIMIENTOS}

Este trabajo se ha realizado mediante la financiación de los proyectos HID98-0323-C05 y REN2001-3438-C07 del Ministerio de Ciencia y Tecnología y PLP/10/FS/97 de la Fundación Séneca de la CARM. Nuestro especial agradecimiento al Area de Coordinación y Aplicaciones Tecnológicas de la D.G.O.H. del Ministerio de Medio Ambiente, al Àrea de Medi Ambient de la Diputació de Barcelona, a la Delegación de Granada de la Consejería de Medio Ambiente de la Junta de Andalucía y a la Agencia Catalana del Aigua por su apoyo.

\section{BIBLIOGRAFÍA}

ALBA-TERCEDOR, J. \& A. SÁNCHEZ-ORTEGA. 1988. Un método rápido y simple para evaluar la calidad biológica de las aguas corrientes basado en el de Hellawell (1978). Limnetica, 4: 51-56.

ALBA-TERCEDOR, J. \& N. PRAT. 1992. Spanish experience in the use of macroinvertebrates as biological 14606 EN-FR, 1992- pollution indicators. In: River Water Quality Ecological Assessment and Control. P. Newman, A. Piavaux \& R. Sweeting (eds): 733-738. Commission of the European Communities, EUR III, Bruselas.

ALBA-TERCEDOR, J. 1996. Macroinvertebrados acuáticos y calidad de las aguas de los ríos. $I V$ Simposio del Agua en Andalucía (SIAGA), Almería, España: 203-213.

ALBA-TERCEDOR, J. \& A. PUJANTE. 2000. Running-water biomonitoring in Spain. Opportunities for a predictive approach. In: Assessing the Biological Quality of Freshwater: RIVPACS and similar techniques. J.F. Wright, D.W. Sutcliffe \& M. Furse (eds.): 207-216. Freshwater Biological Association.

ALBA-TERCEDOR, J., P. JÁIMEZ-CUÉLLAR, M. ÁlVAREZ, J. AVILÉS, N. BONADA, J. CASAS, A. MELLADO, M. ORTEGA, I. PARDO, N PRAT, M. RIERADEVALL, S. ROBLES, C SÁINZ-CANTERO, A. SÁNCHEZ-ORTEGA, M L. SUÁREZ, M. TORO, M. R. VIDAL-ABARCA, S. VIVAS \& C. ZAMORA-MUÑOZ. 2002. Caracterización de cuencas mediterráneas españolas en base al índice español SBMWP como paso 
previo al establecimiento del estado ecológico de sus cursos de agua. Libro de Resúmenes del XI Congreso de la Asoc. Esp. Limnología. Madrid, España.

ARMITAGE, P. D., D. MOSS, J. F. WRIGHT \& M. T. FURSE. 1983. The performance of a new biological water quality score system based on macroinvertebrates over a wide range of unpolluted running-water sites. Water Research, 17: 333-347.

BARBOUR, M. T., J. GERRITSEN, G. E. GRIFFITH, R. FRYDENBORG, E. MCCARRON, J. S. WHITE \& M. L. BASTIAN. 1996. A framework for biological criteria for Florida streams using benthic macroinvertebrates. J. N. Am. Benthol. Soc., 15: 185-211.

BARBOUR, M. T., J. GERRITSEN, B. D. SNYDER \& J. B. STRIBLING. 1999. Rapid Bioassessment Protocols for Use in Streams and Wadeable Rivers: Periphyton, Benthic Macroinvertebrates and Fish, $2^{\text {nd }}$ ed. EPA 841-B-99-002. US EPA, Office of Water, Washington D.C., USA.

BONADA, N., N. PRAT, A. MUNNÉ, M. PLANS, C. SOLÀ, M. ÁLVAREZ, I. PARDO, G. MOYÀ, G. RAMON, M. TORO, S. ROBLES, J. AVILÉS, M. L. SUÁREZ, M. R. VIDAL-ABARCA, A. MELLADO, J. L. MORENO, C. GUERRERO, S. VIVAS, M. ORTEGA, J. CASAS, A. SÁNCHEZORTEGA, P. JÁIMEZ-CUÉLLAR \& J. ALBATERCEDOR. 2002 - a. Intercalibración de la metodología GUADALMED. Selección de un protocolo de muestreo para la determinación del estado ecológico de los ríos mediterráneos. Limnetica, 21: 13-33.

BONADA, N., N. PRAT, A. MUNNÉ, M. RIERADEVALL，J. ALBA-TERCEDOR， M. ÁlVAREZ, J. AVILÉS, J. CASAS, P. JÁIMEZCUÉlLAR, A. MELLADO, G. MOYÀ, I. PARDO, S. ROBLES, G. RAMON, M. L. SUÁREZ, M. TORO, M. R. VIDAL-ABARCA, S. VIVAS \& C. ZAMORA-MUÑOZ. 2002 - b. Ensayo de una tipología de las cuencas mediterráneas del proyecto GUADALMED siguiendo las directrices de la Directiva Marco del Agua. Limnetica, 21: 77-98.

BONADA, N., N. PRAT, A. MUNNÉ, M. RIERADEVALL，J. ALBA-TERCEDOR， M. ÁlVAREZ, J. AVILÉS, J. CASAS, P. JÁIMEZCUÉlLAR, A. MELLADO, G. MOYÁ, I. PARDO, S. ROBLES, G. RAMÓN, M.L. SUÁREZ, M. TORO, M. R. VIDAL-ABARCA, S. VIVAS \& C. ZAMORA-MUÑOZ. 2002 - c.
Criterios para la selección de condiciones de referencia en los ríos mediterráneos. Resultados del proyecto GUADALMED. Limnetica, 21: 99-114.

DIGBY， P.G.N. \& R.A. KEMPTON. 1987 Multivariate Analysis of Ecological Communities. London: Chapman \& Hall.

DIXON, W.J. \& R. JENNRICH. 1983. Stepwise regression. In: BMDP Statistical Software. W.J. Dixon (ed.): 251-263. University of California Press, Berkeley, Los Angeles, London.

D.O.C.E. 2000. Directiva 2000/60/CE del Parlamento Europeo y del Consejo de 23 de octubre de 2000 por la que se establece un marco comunitario de actuación en el ámbito de la politica de aguas. D.O.C.E. L 327 de 22.12.00. 69 pp.

MOSS, D., M.T. FURSE, J.F. WRIGHT \& P.D. ARMITAGE. 1987 The prediction of the macroinvertebrate fauna of unpolluted running-water sites in Great Britain using environmental data. Freshwat. Biol., 17, 41-52.

MUNNÉ, A., C. SOLÀ \& N. PRAT. 1998. QBR: Un índice rápido para la evaluación de la calidad de los ecosistemas de ribera. Tecnología del Agua, 175: 20-37.

ORTIZ, J.L. (2004). La Directiva Marco del Agua: Aspectos relevantes para el proyecto GUADALMED. Limnetica, 22: 5-12.

PARDO, I., M. ÁlVAREZ, J. CASAS, J.L. MORENO, S. VIVAS, N. BONADA, J. ALBATERCEDOR, P. JÁIMEZ-CUÉLLAR, G. MOYÀ, N. PRAT, S. ROBLES, M. L. SUÁREZ, M. TORO \& M. R. VIDAL-ABARCA. 2002. El hábitat de los ríos mediterráneos. Diseño de un índice de diversidad de hàbitat. Limnetica, 21: 115-132.

PRAT, N., A. MUNNÉ, M. RIERADEVALL, C. SOLÀ \& N. BONADA. 2001. ECOSTRIMED. Protocolo para determinar el estado ecológico de los ríos mediterráneos. Barcelona: Diputació de Barcelona, Àrea de Medi Ambient.

REYNOLDSON, T. B. \& J. F. WRIGHT. 2000. The reference condition: problem and solution. In: Assessing the Biological Quality of Freshwater: RIVPACS and similar techniques. J.F. Wright, D.W. Sutcliffe \& M. Furse (eds.): 293-303. Freshwater Biological Association.

RICO, E., A. RALLO, M. A. SEVILLANO \& M. L. ARRETXE. 1992. Comparison of several biological indices based on macroinvertebrate benthic community for assessment of running water quality. Annls. Limnol. 28: 147-156. 
ROBLES, S., M. TORO, C. NUÑO, J. AVILÉS, J. ALBA-TERCEDOR, M. ÁLVAREZ, N. BONADA, J. CASAS, P. JÁIMEZ-CUÉLLAR, A. MELLADO, A. MUNNÉ, I. PARDO, N. PRAT, M. L. SUÁREZ, M. R. VIDAL-ABARCA, S. VIVAS, G. MOYÁ \& G. RAMON. 2002. Descripción de las cuencas mediterráneas seleccionadas en el proyecto GUADALMED. Limnetica, 21: 35-61.

RODRÍGUEZ, P. \& J.F. WRIGHT. 1991. Description and evaluation of a sampling strategy for macroinvertebrate communities in Basque rivers (Spain). Hydrobiologia, 213: 113-124.

SUÁREZ, M. L., M. R. VIDAL-ABARCA, M.M. SÁNCHEZ-MONTOYA, J. ALBA-TERCEDOR, M. ÁlVAREZ, J. AVILÉS, N. BONADA, J. CASAS, P. JÁIMEZ-CUÉLLAR, A. MUNNÉ, I. PARDO, N. PRAT, M. RIERADEVALL, M. J. SALINAS, M. TORO \& S. VIVAS. 2002. Las riberas de los ríos mediterráneos y su calidad: El uso del índice QBR. Limnetica, 21: 63-75.

TORO, M., S. ROBLES, J. AVILÉS, C. NUÑO, S. VIVAS, N. BONADA, N. PRAT, J. ALBATERCEDOR, J. CASAS, C. GUERRERO, P. JÁIMEZ-CUÉLLAR, J. L. MORENO, G. MOYÁ, G. RAMON, M. L. SUÁREZ, M. R. VIDALABARCA, M. ÁlVAREZ \& I. PARDO. 2002.
Calidad de las aguas de los ríos mediterráneos del proyecto GUADALMED. Limnetica, 21: 149-173.

VIVAS, S., J. CASAS, I. PARDO, S. ROBLES, N. BONADA, A. MELLADO, N. PRAT, J. ALBATERCEDOR, M. ÁlVAREZ, M. M. BAYO, P. JÁIMEZ-CUÉLLAR, M. L. SUÁREZ, M. TORO, M. R. VIDAL-ABARCA, C. ZAMORA-MUÑOZ, N. PRAT \& G. MOYÀ. 2002. Aproximación multivariante en la exploración de la tolerancia ambiental de las familias de macroinvertebrados de los ríos mediterráneos del proyecto GUADALMED. Limnetica, 21: 149-173.

WRIGHT J. F., P. D. ARMITAGE, M. T. FURSE \& D. MOSS. 1988. A new approach to the biological surveillance of river quality using macroinvertebrates. Verh. Internat. Verein. Limnol., 23: 1548-1552.

WRIGHT J. F., P. D. ARMITAGE, M. T. FURSE \& D. MOSS. 1989. Prediction of invertebrate communities using stream measurements. Regul. Rivers: Res. Manage., 4: 147-155.

ZAMORA-MUÑOZ, C., C. E. SÁINZ-CANTERO, A. SÁNCHEZ-ORTEGA \& J. ALBA-TERCEDOR. 1995. Are biological indices BMWP' and ASPT' and their significance regarding water quality seasonally dependent? Factors explaining their variations. Water Research, 29: 285-290. 